\title{
Téoros
}

Revue de recherche en tourisme

\section{Le phénomène des gîtes touristiques en Estrie}

\section{Nadia Couture et Réal Viens}

Volume 16, numéro 1, printemps 1997

La région touristique de l’Estrie / Cantons-de-l'Est

URI : https://id.erudit.org/iderudit/1074925ar

DOI : https://doi.org/10.7202/1074925ar

Aller au sommaire du numéro

Éditeur(s)

Université du Québec à Montréal

ISSN

0712-8657 (imprimé)

1923-2705 (numérique)

Découvrir la revue

Citer cet article

Couture, N. \& Viens, R. (1997). Le phénomène des gîtes touristiques en Estrie. Téoros, 16(1), 32-37. https://doi.org/10.7202/1074925ar d'utilisation que vous pouvez consulter en ligne.

https://apropos.erudit.org/fr/usagers/politique-dutilisation/ 


\title{
$+2$. \\ LE PHÉNOMÈNE DES GîTES TOURISTIQUES EN ESTRIE
}

\author{
Nadia Couture, géographe et étudiante à la maítrise \\ en géographie touristique \\ Université de Sherbrooke \\ Réal Viens, géographe \\ Propriétaire d'un gîte touristique à Magog
}

Absent du paysage québécois il y a 20 ans et considéré comme un produit marginal encore à la fin des années 80. I"émergence des gîtes fouristiques a connue une expansion sans précédent depuis sept ans. C'est un portrait de la simation que nous voulons présenter ici à travers la région de l'Estrie qui a connu we evolution très rapide à cet egard. Nous présenterons donc ici un bref historique du produit, ainsi que le profil des gûtes touristiques, des propriétaires et de la clientèle, de même que la perception et la satisfaction de cette dernière. De plus, nous procederons à ane évaluation du chiffre d'affaire total approximatif de ce phénomène dans l'industrie touristique québécoise.

\section{HISTORIQUE}

Rappelons d'abord que c'est en Angleterre que le phénomène des Bed and Breakfast a pris naissance et cela depuis aussi longtemps que les déplacements l'ont obligé et que le gîte était requis dans les petits villages dépourvus d'hébergement commercial. Il a fallu attendre le début des années 80 pour assister aux premiers balbutiements de ce produit au Québec. Et c'est plus précisément à la Fédération des Agricotours du Québec (FAQ) que nous devons d'avoir créé une appellation purement québécoise, ales gîtes du passant $w$. En effet, la fin des années 70 et le début des années 80 ont donné naissance à tout un réseau de fermes d'hébergement à travers le Québec sous l'égide de la FAQ, elle-même constituée par des Agricotours locaux (ces organismes relevaient des bureaux de I'Union des producteurs agricoles (UPA) dans chaque région et veillaient au maintien du réseau régional et à sa qualité de concert avec le Ministère de l'Agriculture, des Pêcheries et de I'Alimentation du Québec (MAPAQ).

C'est donc suite à des demandes d' waccréditation* provenant de personnes ne possédant pas de ferme, ou d'anciens producteurs agricoles retraites que l'on considéra l'éventualité d'offrir un nouveau produit d'hébergement. Ces amaisonsw étaient situées dans des villages, quelquefois à proximité de fermes ou carrément en milieu urbanisé. Afin de distinguer ce produit des fermes d'hébergement on trouva alors $I^{\dagger}$ appellation aGîte du passantw. La FAQ de l'époque etait loin de se douter qu'en lançant ce produit, elle allait donner naissance à autant de rejetons. En effet, nous avons eu droit depuis ce temps à nombre d'appellations telles que, cafe-conette, le gite et le couvert, café-dodo, chambre d'hôte, etc. Le Ministère a finalement légiféré en 1991 et a adopté le vocable "Gite touristiques, mais incluant les gittes de 4 et 5 chambres seulement. Nous savons au= jourd'hui tout ce que cela a causé comme complications autant aux Associations touristiques régionales (ATR) pour les regrouper et les vendre qu'au Ministère lui-même pour les réglementer. Sans parler des autres acteurs de I'hébergement tels que les hôteliers qui n'ont cessé depuis quelques années de réclamer une réglementation touchant tous les gîtes (surtout les 1, 2 et 3 chambres) les accusant de aconcurrence déloyales.

\section{ÉVOLUTION DU PRODUIT}

Comme tout produit de consommation courante, le gite touristique évolue. Perçu d'abord conme un hébergement marginal et bon marché attirant tout juste $1.4 \%$ de la clientèle totale en $1987^{\prime}$. les données de la Corporation Touristique Magog-Orford nous révèle que c'est $6,6 \%$ de la clientèle de cette région qui utilisait ce type d'hébergement à l'été 1994. On peut facilement extrapoler cette constatation aux principales régions touristiques. En moins de 10 ans, nous avons donc assisté à une augmentation de plus du triple. Malgré cette fulgurante popularité, le ministère du Tourisme enregistrait officiellement à la Direction des établissements touristiques 207 émissions de permis pour des gîtes en 1995 et 373 en 1997. En faisant le calcul du nombre de gites inscrits dans les guides touristiques au Québec en 1996, nous en obtenons plus de 1000. Rappelons que la Direction des êtablissements touristiques émet des permis pour des gites de 4 et 5 chambres tandis que les ATR publicisent les gites qui en deviennent membre, donc sans égard au nombre de chambres. 
Selon plusieurs observateurs, le nombre réel de gîtes au Québec se situerait à plus de 1500 et certains avancent même 2000 .

\section{UNE HIÉRARCHIE OBLIGÉE}

Selon la Professional Association of Innkeepers International (PAII) installée aux Etats-Unis, malgré l'appellation générale de Bed and Breakfast, une certaine hiérarchisation s'est installée et a finalement êté définie. Selon nos observations, et cela malgré le manque de règlement et l'absence de regroupement, mis à part la FAQ, cette même hiérarchisation semble vouloir s'installer au Québec. En effet, toujours selon PAII, il $\mathrm{y}$ a trois types de Bed and Breakfast qui se rapprochent d'ailleurs des types de gîtes que nous avons au Québec. Il y a d'abord le Homesray ou le Host Home:

aThis type of establishment is an owneroccupied private home where the buisness of paying guests is secondary to its use as a private residence. The hosts are primarily interested in meeting new people and making some additional monies while continuing their present employment or retirement. Fequently located in residentiel areas, zoning or other government restrictions may prevent the use of signs, public advertising, etc. Usually between 1-3 rooms, these homes are often a member of, and usually inspected by a reservation service organization (RSO) but are rarely required to be licensed or inspected by local applicable governmental agencies .

Ce type d'établissement ressemble à bon nombre de gittes et se rapproche sûrement du chiffre d'affaire moyen actuel, soit autour de $15592,50 \$$.

D'autre part, il y a Egalement le type $B e d$ and Breakfast:

*Formerly a single family dwelling usually in the 4-5-rooms range, this owneroccupied establishment has an equally mixed use as home and lodging with lodging superseding home more often than not. It is located in al legally zoned area and meets all the tax, fire, building and health requirements for this size and use of property. This establishment advertises publicly and can legally post a sign. Like the homestay or host home, because of its size, these B\&Bs usually cannot support a family unit, so the B\&B is often one partner's job and the other has outside income. Often the property is purchased specifically to be a B\&B, but many are converted family homes. Reservations may be made directly with the propertys.

Plus récemment et avec l'évolution du produit québécois, nous rencontrons fréquemment ce type de gîtes surtout dans les grands centres et les pôles tourisliques.

Finalement, le PAII définit et différencie le $B \& B$ du Bed and Breakfast Inn de cette façon :

«Generally small, owner-operated businesses providing the primary financial support of the owner. Usually the owner lives on premises. The building's primary usage is for business. Inns advertise, have business licenses, produce their own brochures, comply with government ordinances, pay all appropriate taxes and post signs. Breakfast is the only meal served and only to overnight guests. The inn may host events such as weddings, small business meetings, etc. Room numbers range from $4-20$ with a small, but increasing number up to 30 . Reservations may be made directly with the property. Note; the distinction between a $\alpha \mathrm{B} \& \mathrm{~B}$. and a $a \& B$ inn is not readily apparent, except with regard to building usagew.

Mis à part le nombre de chambres, puisque l'appellation québécoise est habituellement auberge, ce type de gîteauberge se retrouve maintenant au Québec et semble en progression.

Ces définitions illustrent bien que le Québec suit les tendances nord-américaines de l'évolution du produit. Parti au début des années 80 avec des gites du passant qui étaient plus des chambres d'hôtes que des gîtes amếnagés et décorés pour accueillir les touristes, les dernières années ont fait place à un nouveau type d'etablissement où tout est orienté en fonction du touriste et où les services et les facilités se multiplient : salle de bain privée, balcon privé, foyer dans la chambre, déjeuner servi à l'extérieur, jardin accessible et aménagé, piscine, nombreuses aires de repos et salons, etc. Il va sans dire que l'on est loin des gites qui génèrent un chiffre d'atfaire annuel de $15592,50 \$^{\circ}$ et que l'on parle beaucoup plus maintenant de 50 , 60 et même $80000 \$$ pour ceux offrant de tels services.

\section{LES GITTES TOURISTIQUES EN ESTRIE}

Les gîtes touristiques de l'Estrie, comme ceux de la plupart des autres régions touristiques québécoises, représentent un phénomène grandissant qui ne cesse de croitre et qui prend une ampleur considerable dans l'économie d'aujourd'hui. Nous sarvons que ce segment de l'hébergement est de plus en plus populaire auprès de nombreuses personnes désirant lancer leur petite entreprise et que l'intérết est nettement marqué par să clientèle touristique. Toutefois, que savonsnous exactement des différentes composantes de ce segment de l'hébergement? Quel type de gîte y a-t-il en Estrie? Quel est son taux d'occupation? Quel est le prix moyen pour une chambre? Quels sont les revenus générés par ces entreprises? Les propriétaires ont-ils un deuxième emploi? S'agit-il pour eux d'un projet de retraite ? Ont-ils une formation en tourisme? Qui est la clientèle? D'où vient-elle? Comment a-t-elle entendu parler des gîtes qu'elle visite? Est-elle satisfaite du produit? Quelle est la structure des gîtes à l'extérieur du Québec? Où nous situons-nous par rapport aux comparables? Etc. Ces questions ne représentent seulement qu'une infime partie des interrogations exprimées par de nombreux intervenants touristiques, fasant maintenant face à ce nouveau phénomène en évolution rapide dans leur région, et aussi par les propriétaires de gîtes, mais qui restent trop souvent sans rếponse.

Puisque cette situation demeure un champs d'analyse bien mal connu sinon inconnu, quelques études ont êté effectuées au cours des deux dernières années. nous permettant maintenant d'obtenir des données essentielles pour bien connaître. comprendre et cerner l'ensemble des gîtes, d'autant plus que la littérature est très limitée, pour ne pas dire inexistante dans le cas des références récentes datant de moins de cinq ans. La suite du texte fait état des principaux résultats obtenus à partir des différentes études sur le sujet. 


\section{LE PROFIL DES GÎTES TOURISTIQUES}

Une étude effectuée à l'été 1995 auprès de 50 propriétaires de gîtes touristiques établis en Estrie a été réalisée par le biais d'un questionnaire lors d'entrevues individuelles et nous a permis d'obtenir des résultats intéressants concernant les gîtes touristiques estriens. Le chiffre en pourcentage, représenté entre parenthèses, indique la proportion des répondants aux questions.

Cette étude démontre que le taux d'occupation du gîte selon les différentes saisons est de $61 \%$ et plus à l'été $(60 \%)$, $10 \%$ aे $50 \%$ au printemps $(78 \%), 10 \%$ à $50 \%$ à l'automne $(68 \%)$ et $10 \%$ aे $50 \%$ at l'hiver $(78 \%)$; l'été est donc l'unique saison qui permet de maintenir un seuil d'occupation très important. D'ailleurs, l'automne connaît un taux d'occupation légèrement plus élevé que l'hiver at le printemps, probablement à cause des activites automnales nombreuses, telles que la cueillette des pommes, la visite des vignobles, la randonnée pédestre et l'accès aux télésièges pour admirer les paysages estriens uniques et colorés.

L'étude nous démontre aussi que $38 \%$ des gîtes offrent des forfaits et parmi ceux-ci, les deux principaux offerts avec l'hébergement sont le souper/table champêtre $(18 \%)$ et le golf $(15 \%)$. Selon la moitié des hôtes, le prix moyen d'une chambre pour une nuit en occupation simple est de $35 \$$. Suivent $24 \%$ des propriétaires qui affirment que le prix moyen, en ce qui les concerne, est de 45s. Compte tenu que le profil des gites de l'étude de 1995 est fort différent de ceux choisis pour participer à l'étude de 1996 (étude de la clientèle que nous verrons plus loin), ce prix moyen de location de chambre se situe en bas de la moyenne régionale observée qui est de $60 \$$ (prix moyen minimum pour 2 personnes, le prix pour une personne varierait de $50 \$$ à $55 \$$ en moyenne). Pour ce qui est du style des gîtes, nous en retrouvons plusieurs en Estrie, dont le plus populaire est le campagnard $(48 \%)$. Le style victorien suit avec $18 \%$, tandis que seulement $8 \%$ des gîtes correspondent à un style moderne. Ajoutons que $40 \%$ des maisons ont eté acheté spécifiquement pour y instaurer un gîte et que $10 \%$ avait déjà cette fonetion.

\section{PROFIL SOCIO-ÉCONOMIQUE DES PROPRIETTAIRES}

Toujours selon l'étude effectuéc à l'été 1995 auprès de 50 propriétaires de gîtes en Estrie, plus de la moitié de ceux-ci (56\%) proviennent de l'extérieur de l'Estrie. Nous constatons alors que la région touristique de l'Estrie est très convoitée par les gens désirant lancer un gitte touristique. L'étude démontre aussi que $46 \%$ des proprietaires ont un emploi régulier et rémunéré autre que celui du gîte, le poste d'enseignant étant occupé par $22 \%$ d'entre eux. Parmi ceux n"ayant pas un autre emploi, $59 \%$ affirment que c'est un projet de retraite. Les gîtes touristiques ayant connu une très forte croissance ces derniètes années, $70 \%$ des proprietaires croient que cette tendance va se poursuivre. Il y a également $70 \%$ des hôtes qui possèdent leur gite depuis moins de 4 ans; cette statistique vient appuyer les données observées plus tôt en regard de l'accélération du nombre de gites de 1987 at 1994.

En ce qui concerne les regroupements de gites touristiques en Estrie, 20 propriétaires sont membres de la FAQ et 18 trouvent que cette association est importante pour leur entreprise, tandis que 2 la considèrent comme étant un peu importante. Selon le Guide des gites du passant en $1996 / 1997^{8}$, la FAQ comptait 394 membres au niveau provincial, dont $27 \mathrm{mem}$ bres provenaient de l'Estrie. D'autre part, $78 \%$ des propriétaires sont membres de l'Association Touristique de 1'Estrie. Parmi ces membres, la majorité $(62 \%)$ estime que leur membership à l'ATE est un apport important pour leur entreprise. $6 \%$ affirment un peu, $6 \%$ répondent non et $4 \%$ ne savent pas. Selon le Guide touristique de l'Estrie". le nombre de gites touristiques de l'Estrie qui en est membre en 1996 est de 93 , dont 14 ì Magog-Orford et II à North Hatley.

\section{PROFIL SOCIO-ÉCONOMIQUE DE LA CLIENTÈLE}

Les résultats obtenus proviennent de l'étude effectuée à l'été $1996^{\circ}$ auprès de 339 clients ayant visité les gites touristiques estriens. La méthodologie employée est la suivante: 100 questionnaires ont été distribués dans 10 gittes touristiques choisis au hasard parmi les 50 de la première étude, afin que les propriétaires

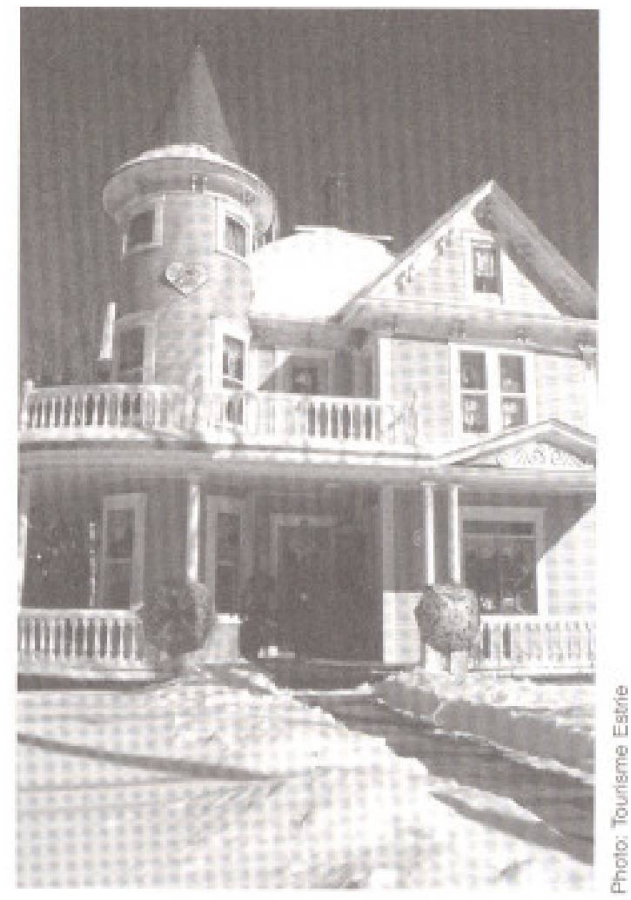

les redistribuent à leur tour à leurs clients. Ensuite, ces derniers devaient nous les retoumer par la poste après les avoir remplis. Les questionnaires n'ayant pas tous été distribués par des propriétaires, nous évaluons done le nombre total à environ 600 avec un taux de réponse effectif d'approximativement $56,5 \%$, soit 339 clients, ce qui est appréciable étant donné les 3 etapes à franchir pour le questionnaire, e'est-à-dire le propriétaire, le client et la poste. Le chiffre en pourcentage, indiqué entre parenthèses, représente la proportion de la clientèle à avoir répondu aux questions.

Tout d'abord, cette étude nous démontre que les visiteurs proviennent principalement du Grand Montréal (Montréal, Montérégic et Laval) avec une proportion de $41 \%$. Nous remarquons d'ailleurs que les gens n'ont parfois pas besoin d'aller bien loin pour se sentir en vacances puisque 16 estriens ( $5 \%$ ) ont visités les gîtes touristiques de leur propre région. D'autre part, près des deux tiers des répondants n'ont aucun enfant à̀ charge (63\%) et la clientèle semble assez jeune avec une proportion de $22 \%$ qui se situent de 18 aे 30 ans et $31 \%$ de 31 à 40 ans (53\% de 18 à 40 ans). Une proportion importante de $29 \%$ se situe aussi dans la tranche des 41 à 50 ans. Plus de la moitié des répondants ont atteint le niveau de scolarité universitaire $(51 \%)$ et possèdent un revenu brut familial annuel de $50001 \$$ 
et plus pour près de la moitié des répondants $(48 \%)$, soit $21 \%$ dans la catégorie de $50001 \$$ à $70000 \$$ et de $27 \%$ dans celle de 70001 s et plus.

\section{PERCEPTION DE LA CLIENTÈLE}

Toujours selon l'étude effectuée à l'été 1996 auprès des 339 clients visitant les gites touristiques de l'Estrie, il est possible de connaître leur perception de ceuxci. Les principaux résultats obtenus nous apprennent que la clientèle a entendu parler du gite visité (qui participe à cette étude) principalement par le Guide touristique de l'Estrie $(28 \%)$ et par leurs parents et amis $(26 \%)$; le Guide, étant distribue gratuitement en 285000 exemplaires à chaque année, est un très bon outil de publicité pour les gîtes comme nous le démontrent ces résultats. De plus, il en est de méme pour le bouche à oreille effectué par les parents et amis puisque cette publicité est perçue comme étant la plus fiable pour les voyageurs. L'autre moyen d'information le plus populaire est le Bureau d'information touristique (BIT) dans une proportion de $20 \%$. En ce qui concerne les principales raisons pour lesquelles le client a choisi ce gîte, e'est d'abord parce qu'il se trouvait sur sa route $(20 \%)$, ensuite pour essayer ce type d'hébergement $(18 \%)$ et pour l'accueil personnel et chaleureux (17\%).

Parmi l'ensemble de la clientèle, près des trois quarts $(73 \%)$ n'en était pas à leur première expérience dans un gite touristique et $38 \%$ du total des clients en ont visité 2 à 4 jusqu'à maintenant. À la lumière de ces résultats, nous pouvons constater que les voyageurs prennent goût à ce type d'hébergement et qu'ils en sont satisfaits puisqu'ils y retournent à maintes reprises. Pour ce qui est de la durée des séjours, $64 \%$ des clients ont passé une nuit tandis que $24 \%$ en ont passé deux. Nous remarquons que le nombre de 2 nuitées est relativement élevé puisqu'autrefois, les gîtes étaient considérés comme un hébergement de circuit et non de destination, $\mathrm{c}^{\dagger}$ est-à-dire que les passants avaient pour habitude d'y sćjourner une seule nuit. Cependant, en comparant le nombre de nuitées avec la moyenne de nuitées dans les hébergements de l'Estrie, 2,6 nuitées", toutes catégories confondues, nous obtenons un nombre plus faible pour les gites touristiques, soit $1,4 \%^{12}$.
En ce qui concerne le prix payé pour une nuit d'hébergement, majoritairement en accupation double, l'étude nous apprend que $45 \%$ des visiteurs ont déboursé $60 \%$ et moins (la classe la plus populaire étant celle de $51 \$$ à $60 \$$ avec une proportion de $30 \%$ ) et que $55 \%$ ont déboursé $61 \$$ et plus (la classe de $81 \$$ à $90 \$$ étant majoritaire avec $31 \%$ ). Ces résultats nous démontrent que le prix des gîtes a considérablement augmenté ces dernières années et les raisons l'expliquant sont sûrement l'augmentation de la qualité du produit et des services offerts, ainsi que le développement touristique de certaines villes ou de certains villages qui ont engendrés de forts achalandages, sans oublier bien sûr l'augmentation du coût de la vie. Pour ce qui est du degré de satisfaction face au gîte visité, il est très satisfaisant dans $48 \%$ des cas et exceptionnel pour $33 \%$ des clients, ses qualités les plus importantes étant l'ambiance générale $(28 \%)$ et l'accueil des hôtes $(20 \%)$.

Nous venons de voir ici que la clientèle semble entièrement satisfaite du gîte touristique visité, particulièrement au niveau de l'ambiance générale et de l'accueil des hôtes. Pourtant, l'étude sur les propriétaires à l'été 1995 nous démontre que $88 \%$ des hôtes n'ont aucune formation dans le domaine du tourisme les ayant préparé à lancer leur entreprise. Alors, même si la plupart des propriétaires n' ont pas de formation en tourisme, les clients sont en grande partie satisfaits de l'accueil reçu par leurs hôtes. Toutefois, pour expliquer cette situation, nous concluons que lors de l'enquête auprès de la clientèle à l'été 1996, nous n'avions pas réalisé que les propriétaires ayant accepté de participer à l'étude étaient probablement ceux qui avaient le meilleur produit à offrir, ce qui nous amène donc à inviter le lecteur à tenir comple de cette mise en garde dans l'interprétation des résultats. découlant de ces recherches.

La principale raison d'utiliser les gites touristiques de préférence aux autres modes d'hébergement est pour l'accueil personnel et chaleureux ( $54 \%)$, une qualité qui ne ressort pas toujours dans l'hôtellerie traditionnelle. Concernant la façon dont la clientèle a entendu parler des différents gîtes visités au Québec, c'est d'abord par leurs parents et amis $(26 \%)$ et ensuite par le Guide touristique de l'Estrie $(23 \%)$. Du côté de la satisfaction générale, les trois qualités les plus importantes d'un gîte selon la clientèle sont l'accueil des hôtes (12I réponses), la qualité de la chambre (102) et le rapport qualité/prix (101). D'autre part, les principaux points faibles retrouvé dans l'ensemble des gîtes visités sont le prix (71 réponses) et la qualité de la chambre (68 réponses). Toutefois, nous constatons qu'il n'y avait aucun point faible dans le cas de 71 réponses ${ }^{13}$. Devenue plus exigeante en matière de confort et de services, la salle de bain privée est primordiale pour $46 \%$ de la clientèle. L'étude démontre d'ailleurs que $34 \%$ de la clientèle a visité des gîtes à l'extérieur du Québec et parmi ces visites, 71 ont été faites en Europe, 51 aux Etats-Unis et 48 au Canada anglais.

\section{DES EXEMPLES DE COMPARAISONS DANS PLUSIEURS PAYS}

Cette étude, effectuée à l'hiver 1995/ $1996^{14}$, examine la structuration des gîtes touristiques hors du Québec à l'aide d'une revue de littérature et de contacts téléphoniques auprès de personnes ressources. En évaluant la situation actuelle québécoise des gîtes touristiques par rapport à ses comparables dans différents pays, on s'aperçoit que le Québec est moins bien structuré. Ce retard est identifié dans plusieurs secteurs tels que l'encadrement, la classification, les associations, l'inspection, la réglementation, les formations et autre.

Pour ne citer que quelques exemples, Ia Grande-Bretagne a mis sur pied un système de classification très détaillếe quí fonctionne avec cinq couronnes pour démontrer le nombre de services offerts et quatre qualificatifs qui expriment le degré de qualité du gîte. Les États-Unis aussi possèdent un système de classification très élaboré qui fonctionnent avec un système de cinq diamants. La France a quant à elle une association nationale très bien structuré et exigeante envers ses membres de la «Fédération Nationale des Gîtes de France* et qui compte 17000 chambres d'hôtes, ce qui repré-sente $28,3 \%$ de l'ensemble de l'hébergement de la Fédération (1500 chambres d'hôtes sont créées à chaque année). Au Nouveau-Brunswick, le permis d'exploitation est obligatoire pour tous les gites touristiques, peu importe le nombre 
de chambres, le prix variant selon le nombre de chambres exploitées dans le gîte. Par cetté étude comparative, nous constâtons qu'il y a plusieurs éléments structurants très interessants qui peuvent s'appliquer à l'échelle québécoise.

\section{PREMIĚRE TENTATIVE D'UNE ÉVALUATION DU CHIFFRE D'AFFAIRES ANNUEL DES GITTES TOURISTIQUES}

Lorsque vient le temps d'estimer l'importance économique des gîtes touristiques, peu de chiffres officiels sont disponibles. En regard du sondage effectue aupres des propriétaires et de la clientèle, il appert que notre evaluation demeurera approximative et ne donnera qu'un ordre de grandeur en attendant des études plus exhaustives.

Dans un marché global de près de 5,0 milliards de dollars pour l'industrie touristique au Québec en 1996, les gîtes semblent ne représenter qu'une goutte dans l'océan. De même, quand on établit la part estrienne des gîtes en terme d'importance économique versus les retombées globales (185 millions de dollars), on constate que l'apport est là aussi peu significatif. On trouvera dans les prochaines lignes l'évaluation du chiffre d'affaire approximatif des gîtes touristiques en Estrie et au Québec.

Afin d'évaluer sommairement le chiffre d'affaire total des gites en Estrie, nous avons décidé de nous baser sur l'étude de 1995 à partir de différentes variables obtenues par les 50 propriétaires de gittes, tels que le nombre moyen de chambres par gîte et le coût moyen d'une chambre pour une nuit. Le tableau 1 vous démontre alors le raisonnement, à partir de ces données et de notre connaissance du marché. pour établir le chiffre d'affaire approximatif des gites touristiques en Estric.

C'est la premiere fois que nous avons un ordre de grandeur sur le revenu brut d'un propriétaire de gite touristique, Certaines personnes travaillant directement en tourisme, avec qui nous avons discuté de ce résultat, l'ont trouvé tout à fait plausible. De plus, notre connaissance du réseau des gites touristiques en Estrie nous fait croire, comme les intervenants touris- tiques, que ce résultat est très près de la réalité.

Même si nous croyons que le chiffre d"affaires approximatif de $19404 \$$ est plausible, il est tout de même plus prudent d'effectuer un deuxième calcul qui permettrait de valider ou pas cette première evaluation. Pour ce faire, ce deuxième calcul concernant le chiffre d'affaires approximatif des gîtes de l'Estrie, présenté au tableau 2, s'est basế sur le Guide touristique de l'Estrie, édition 1996.

Ce deuxième calcul ayant êté effectué, nous pouvons confirmer que le chiffre d'affaires moyen d'un propriétaire estrien se situe aux alentours de 20000 \% puisque les deux résultats obtenus par les deux calculs sont très similaires. C'est done un chiffre d'affaires de près de 2 millions de dollars que génèrent les 93 gites membres de l'A.T.E. en 1996. Si on tient compte de tous les gîtes touristiques que l'on peut trouver en région, ce serait alors 2.5 à 3 millions de dollars dont il serait question.

En ce qui concerne l'évaluation du chiffre d'affaire approximatif des gites touristiques pour l'ensemble du Québec, nous nous permettrons ici de faire une extrapolation à partir des résultats obtenus d̀ l'aide du guide touristique de l'Estrie. Plusieurs t́valuent le nombre total de gîtes au Québec à 1500 et mểme $2000^{1 k}$. Ainsi, un peu simplement, nous multiplierons la moyenne par gite obtenue en Estrie par le nombre de gîtes xprésumés au Québec. Le tableau 3 nous donne ainsi une idée du chiffre d'affaires total des gîtes au Québec en 1996.

Il va sans dire que cette première évaluation est plus que sommaire puisque ce chilfre est très conservateur. II n'est pas impossible que l'innpact réel atteigne les 50 millions de dollars. Ce nombre de 1500 gites est un strict minimum. Toutefois, ces revenus nous donne une indication sur l'importance et l'ampleur du phénomène des gîtes touristiques dans l'économic québécoise.

\section{TENDANCES...}

Les tendances actuelles des années 90 semblent être, pour plusieurs entreprises, reliees au phenomene du cocooning, le syndrome de rester chez soi, comme le définit Faith Popcorn. Ce qui correspond
TABLEAU 1

Chiffere d'affaire approximatif des gîtes touristiques. participant a Petude des proprictaires en Estrie (1995)

- Nombre de gites participant à rétude = 50

- Nombre moven de chambres par gite $=174$ chambres au total / 50 gites $=3,5$ chambres

- Prix de lacation moyen = 465 en occupation simple, donc environ 563 en occupation double

Supposons ici gussi une ouwerture annualle Equivalente a 11 mois (1 mais de vacances]

- 11 mois * 30 jours au nuitées dispanibles = 390 jours nu nuitées dispanibles

- $330 * 3,5$ chambres $=1155$ nuitées totales disponibles

- Taux di becupation de 30\% (moyenne des 4 saisons) affecté है 1155 nuitedes $=346.5$ nuitées

- 346,5 nuitées* 565 pour une nuit $=19404,005$

Revenu total moven d̛ un gîte ex Estrie (1945)

TABLEAU 2
Chilfre of affaire approximatif des gites touristiques membres de I'ATE en 1996

- Nombre de gîtes en Estrie en 1996

|salna guide touristiquel $=96$

- Nombre moyen de chambres gar gĭte $=327$ chambres au total / gs gites $=3,5$ chambres

- Prix de location annuel le plus bas (moyenne) = 60,065

Supposans une ouverture annurelle equivalenta à 11 mois (1 mois de vacances)

- 11 mois * 30 jours a 300 jours ou nuitées disponibles

- $300 \cdot 3.5$ chambres $=1155$ nuitées totales annuelles possibles

- Taux d'accupation de $30 \%$ affecté a 1155 nuites $=$ 346,5 nuitées

- 346,5 nuitées " 60.065 pour une nuit = 20810,795 Revenu total d'ua gite en Estrie (1996)

- 20 280,79S * 93 qites $=1935403,475=$

Revenus totaux des gîtes membre de I'ATE

\begin{tabular}{c} 
TABLEAU 3 \\
$\begin{array}{c}\text { Chiffe d'aflaire approximatif des gittes touristiques } \\
\text { au Quebec en } 1996\end{array}$ \\
\hline $20810.795+1500$ gîtes $=312161855$ \\
\hline
\end{tabular}

tout à fait au concept d'hébergement du gite touristique. Les clients veulent se sentir chez eux. mais ailleurs dans un hébergement de grand confort et de qualité. D'autant plus que plus il y a des services offerts dans le gite, et plus la clientèle sera enchantée de pouvoir rester sur place ou à proximité de son lieu d'hébergement. Selon Faith Popcorn ${ }^{14}$, aLe cocooning ne désigne plus seulement un endroit qui serait le foyer mais plutôt un état d'esprit: l'autosauvegarde. Nous voulons nous sentir en securité partout où nous allons. Nous voulons que le cocon nous accompagne en voyage. ...le nombre de gites ruraux s'ouvrant un peu partout dans le pays (aux Etats-Unis) monte en fleche: près de 12000 en 1989 alors qu'il y en avait à peine 2000 dix ans plus tôt . La tendance du cocooning est là pour rester longtemps. 
Quant aux prévisions pour les prochaines annees, elles s'avèrent selon nous très prometteuses. La variété des differrents gîtes continuera à plaire aux voyageurs. Certains auront plus de difficulté à survivre étant donné le nombre important de gîtes touristiques; le client aura amplement le choix de son hébergement, il choisira donc un gîte où les services seront les plus personnalisés et cela, probablement pour le même prix que pour un gîte qui n'en possède pas. Si nous jetons un coup d'oeil sur les pays plus avancés dans la structure des gîtes touristiques, nous remarquons que le nombre total de gites ne fait que prendre de l'expansion à chaque année; alors puisque nous avons du retard sur eux, et que nous semblons suivre leurs tendances, il est normal que les gites soient là encore pour un bon nombre d'années. Alors pour ceux n'ayant pas encore tenté l'expérience dans un gîte touristique estrien chaleureux, vous aurez suffisamment de temps de l'essayer !

\section{NOTES}

I MINISTEREE DU LOISIR. DE LA CHASSE ET DE LA PECCHE (1987) Enude sur les réseaux d"hébergement, volume 1: volet: auberges de jeunesse, gites du passant el fermes de vacances. Service de l'analyse et de la recherche socio-économique, Québec, avril. 154 p.

2 CORPORATION TOURISTIQUE MAGOG-ORFORD (1994) Evaluation de la répartition de Thébergement dans la station tourisitque Magog-Orford. Enquête interne, septembre. $15 \mathrm{p}$.

3 TOURISME QUÉBEC (1997), op. orale

4 PROFESSIONAL ASSOCIATION OF INNKEEPERS INTERNATIONAL: the North American association for B\&B/country inns. Adresse Internet: htip://www.paii. org/definitions.html., 1997

5 COUTURE, Nadia (1997) Les gites wowistiques en Extrie: wn phenomène ent entergence rapide. Mémoire de maitrise, en préparation, Université de Sherbrooke, Département de géographie et téléddétection. Sherbrooke.

6 lbid.

7 laid

8 FÉDERATION DES AGRICOTOURS DU QUÉBEC (1996) Gites du passant au Quebec. Montreal, Editions Ulysse, $269 \mathrm{p}$.

9 TOURISME ESTRIE ET TOURISME QUÉBEC (1996) Guide tourisique de l'Es:trie 1996/7997. Québec, ge édition, 141 p.

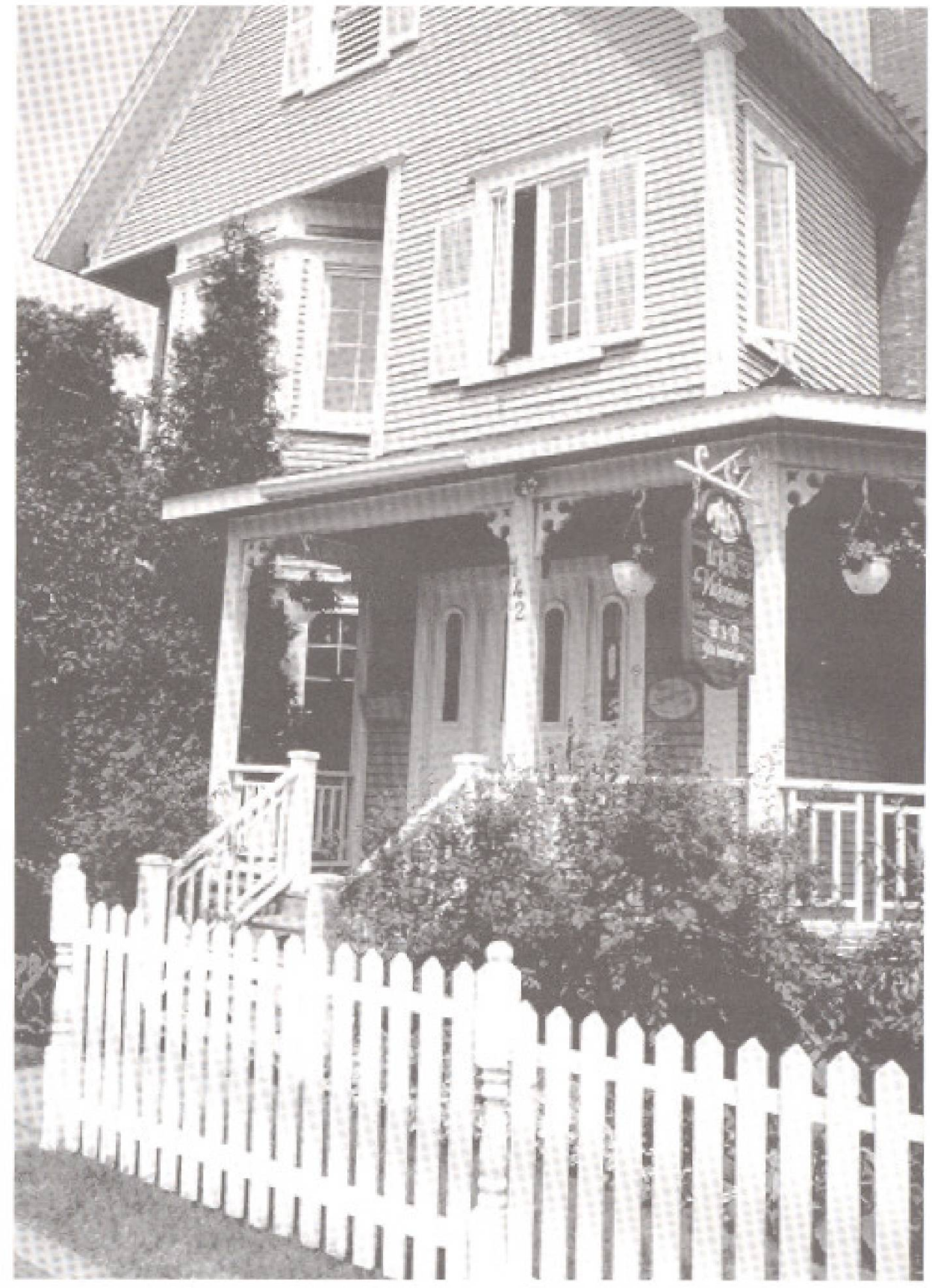

10 COUTURE, Nadia (1997) op. cit.

11 TOURISME QUEBEC (1996) Les touristes québécois au Québec en 1994. Annexe stattistique, juin, $181 \mathrm{p}$,

12 CORPORATION TOURISTIQUE MAGOG-ORFORD (1994) op. cit.

13 Ces chiffres representent le nombre total de réponses car les répondants pouvaient cocher plus d'un élémeni.

14 COUTURE, Nadia (1997) op. cit.

15 S il y a 93 gites touristiques membres de I'ATE en 1996, il y en a sans doute encore plus qui n'en sont pas membres, ce qui signifie que les revenus totaux obtenus isi sont un strict minimum.
16 Ce ehiffre a été obuenu en additionnant le prix de la chambre la moins chere de chaque gîte pour l'annse 1996. Le total a été divisé par le nombre de gites (93) pour en arriver à une moyenne de 60.065 .

17 Ce taux a été pris a partir des chiffres du Ministère du Tourisme 1993 et qui évaluent a $31 \%$ le taux d'occupation des etablissements de 5 aे 39 chambres.

18 CHOUINARD. Marie-Andrée (1996) aGrogne dans l'hótelleries, Le Devoir, no 165.17 juillet, p. A2

19 Popcorn, Fath $(1991)$ Le Fappon Popcom comnent vivmons-nous lian 2000 ? Ed. De l'homme, $268 \mathrm{p}$. 